\title{
Supporting Documentation
}

\section{Assessing Sites Contaminated with Unexploded Ordnance: Statistical Modeling of Ordnance Spatial Distribution}

\author{
JACQUELINE A. MACDONALD \\ MITCHELL J. SMALL
}

In the main article, we describe three functions used to evaluate whether a given statistical model fits a spatial data set. We explain one of the functions, known as $K(h)$, in the article. Here, we provide additional information about the other two functions, $G(h)$ and $F(h)$. We also document that both functions indicate that the cluster model is consistent with the two data sets described in the paper, while the complete spatial randomness (CSR) model is not.

Recall from the main paper that $G(h)$ represents the cumulative probability distribution function of the distances between each event in the data set and its nearest neighbor. For the CSR model (see references 16 and 17 in the main text),

$$
G(h)=1-\exp \left(-\lambda \pi h^{2}\right)
$$

where $\lambda$ is the total number of events divided by the total area. For the cluster model, Diggle (17) and Kaluzny et al. (24) recommend determining $G(h)$ by averaging the $G(h)$ values for a large number of model simulations. For each simulation, $G(h)$ is determined from the following estimator:

$$
\hat{G}(h)=n^{-1} \times(\text { number of interevent distances less than or equal to } h)
$$

where $n$ is the total number of simulated events (199 for Fort Ord and 805 for Tobyhanna).

As explained in the main article, $F(h)$ represents the cumulative probability distribution of distances from each member of a grid of regularly spaced points to the nearest event. For the CSR model, this function follows equation (1) (with $G$ replaced by $F$ on the left-hand side). The expected values for the cluster model are determined by simulation, as for $G(h)$. 
Figures S-1 and S-2 compare the functions $G(h)$ and $F(h)$, respectively, for the Fort Ord data against the values expected for the CSR and cluster models. Figures S-3 and S-4 show the same analyses for the Tobyhanna data set. For example, the top half of Figure S-1 plots $G(h)$ computed from the Fort Ord data as a function of the values of $G(h)$ one would expect if the cluster model fit the data. If the cluster model fit were perfect, this plot would result in a straight line from the origin to the upper right corner. For a reasonable model fit, the data should be contained within the $98 \%$ confidence interval shown on the plot. This $98 \%$ confidence interval was determined by simulating the CSR model 100 times and plotting the upper $99 \%$ and lower $1 \%$ of the simulated values for $G(h)$ as a function of the theoretical $G(h)$ (equation 1). As shown, the Fort Ord data are outside this $98 \%$ confidence interval, indicating that the CSR model does not fit the data. The lower half of the figure, in contrast, shows the evaluation of the cluster model fit according to the $G$ function. Instead of plotting the data as a function of the expected value of the CSR model, in this case the data are plotted as a function of the expected value of the cluster model, estimated from the average of 100 simulations. Since the horizontal axis in this case represents the cluster model, not the CSR model, the plot of data against this axis is different from that for the top part of the figure. The results show that for Fort Ord, the data are almost entirely contained within the $98 \%$ confidence interval for the cluster model. The small excursion outside the confidence interval at the lower left corner is likely a result of errors in reporting UXO locations. The spatial modeling techniques we use here assume that events are not colocated. In a few cases, the Fort Ord database reported identical geographic coordinates for more than one UXO item, likely due to crews excavating large holes and being unable to precisely locate the original coordinates of each retrieved item prior to excavation.

All four figures show that the cluster model (with different parameters for Fort Ord and Tobyhanna) is consistent with the data at both Fort Ord and Tobyhanna, but the CSR model is not. 


\section{Evaluation of CSR Model Fit Using G}

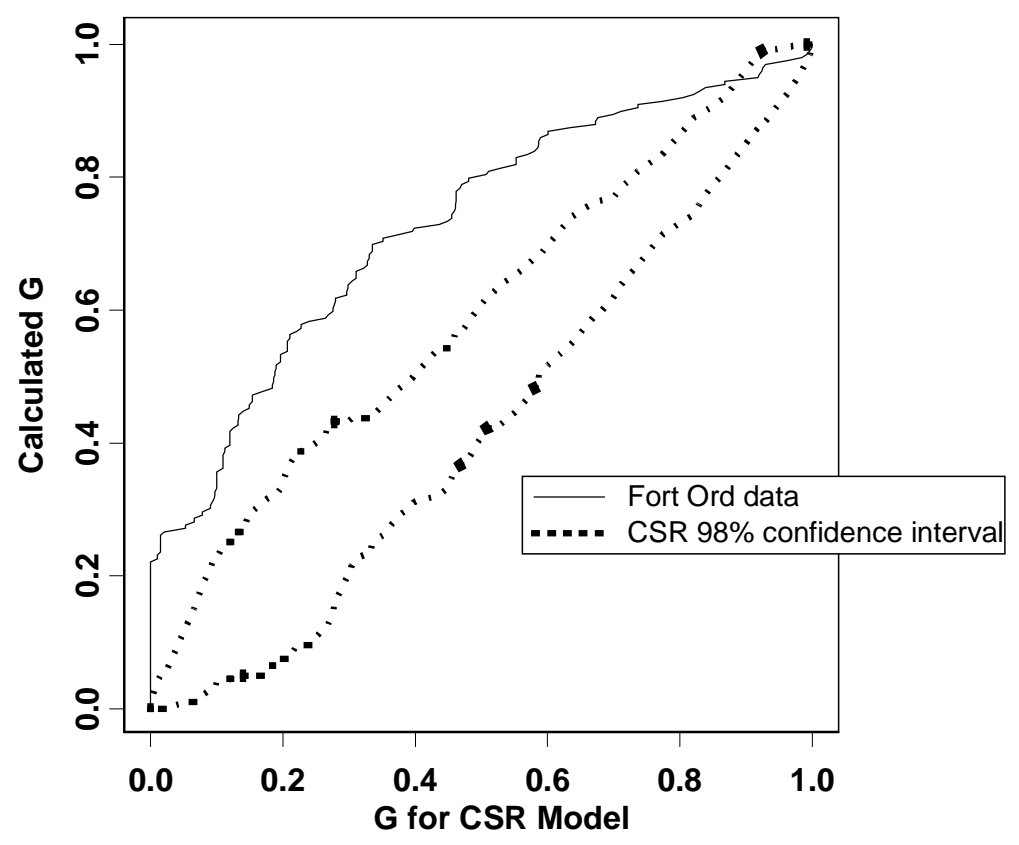

Evaluation of Cluster Model Fit Using G

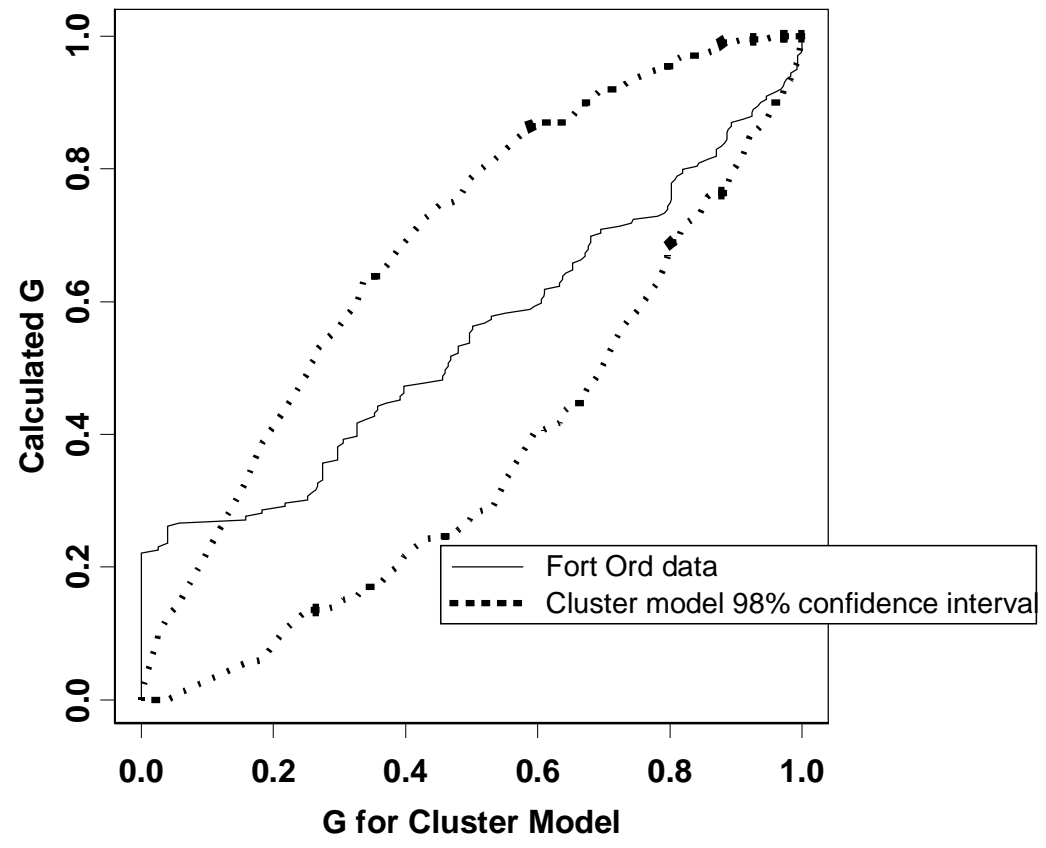

Figure S-1: Comparison of CSR (upper graph) and cluster (lower graph) model fits to the Fort Ord UXO data using the distribution function $G$. The graphs plot the empirical data against the expected value of $G$ if the model holds. As shown, the empirical plot (upper chart) is outside the $98 \%$ confidence interval for the CSR model but almost entirely within this confidence interval for the cluster model (lower plot). 


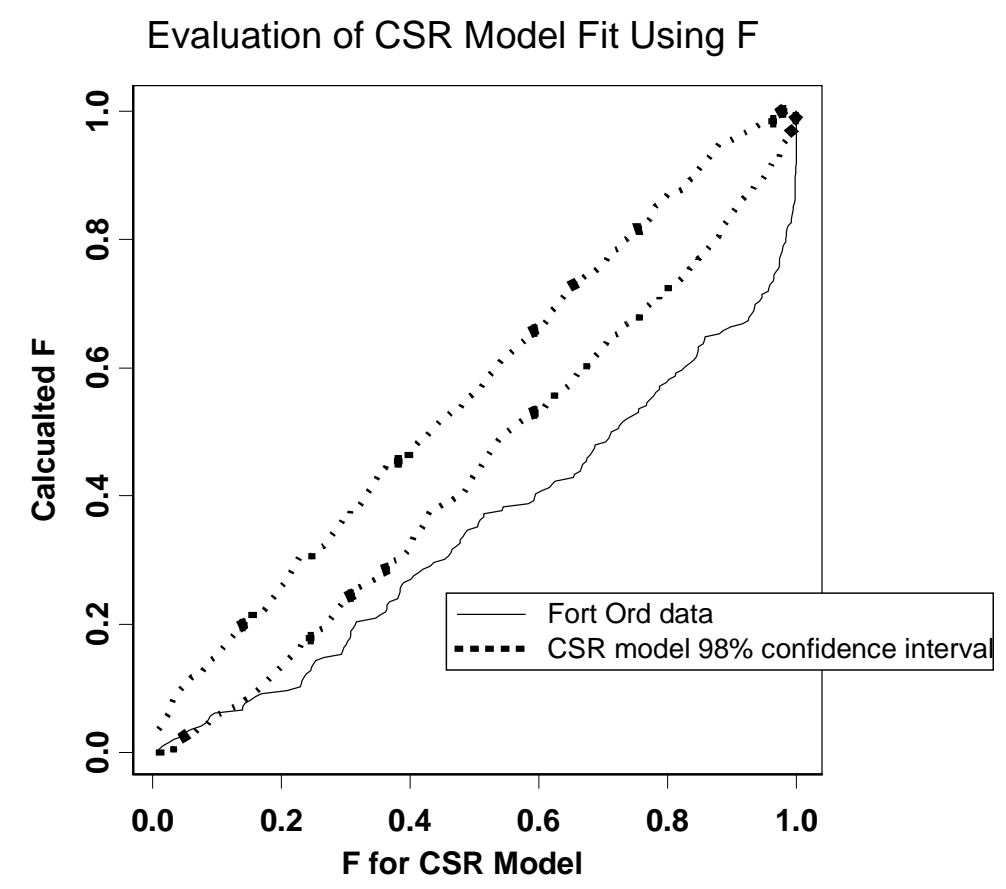

Evaluation of Cluster Model Fit Using F

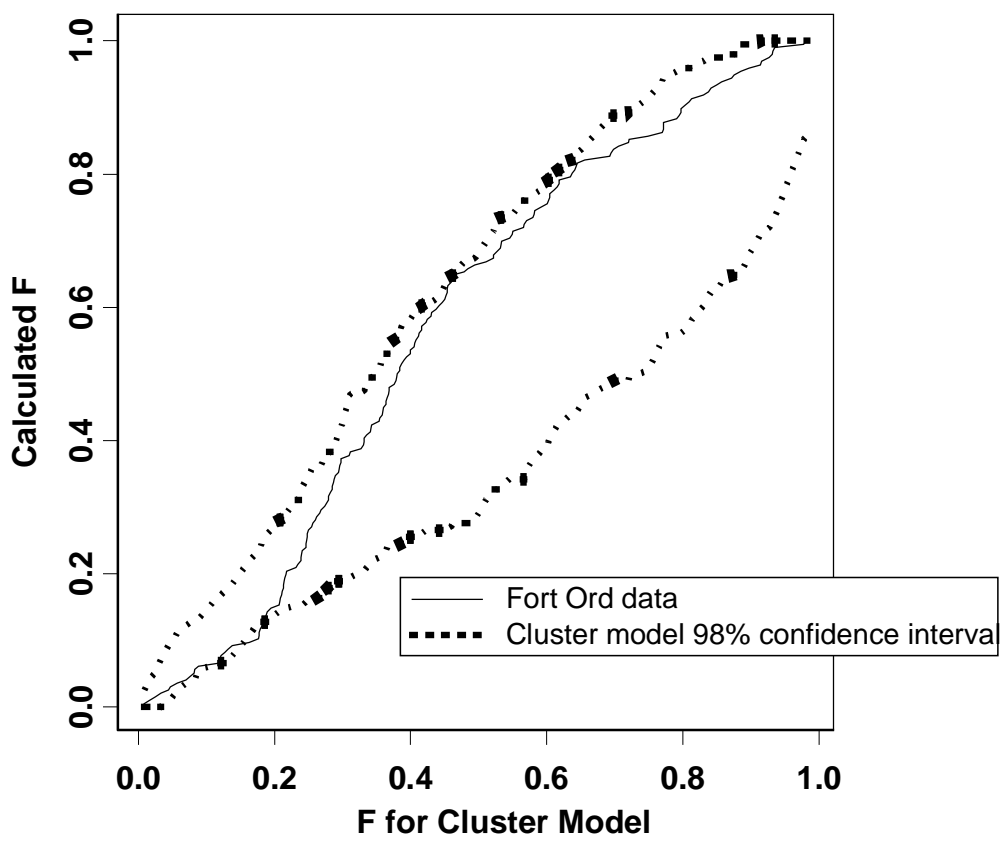

Figure S-2: Comparison of CSR (upper graph) and cluster (lower graph) model fits to the Fort Ord UXO data using the distribution function $F$. The empirical plot (upper chart) is outside the $98 \%$ confidence interval for the CSR model but almost entirely within this confidence interval for the cluster model (lower plot). 


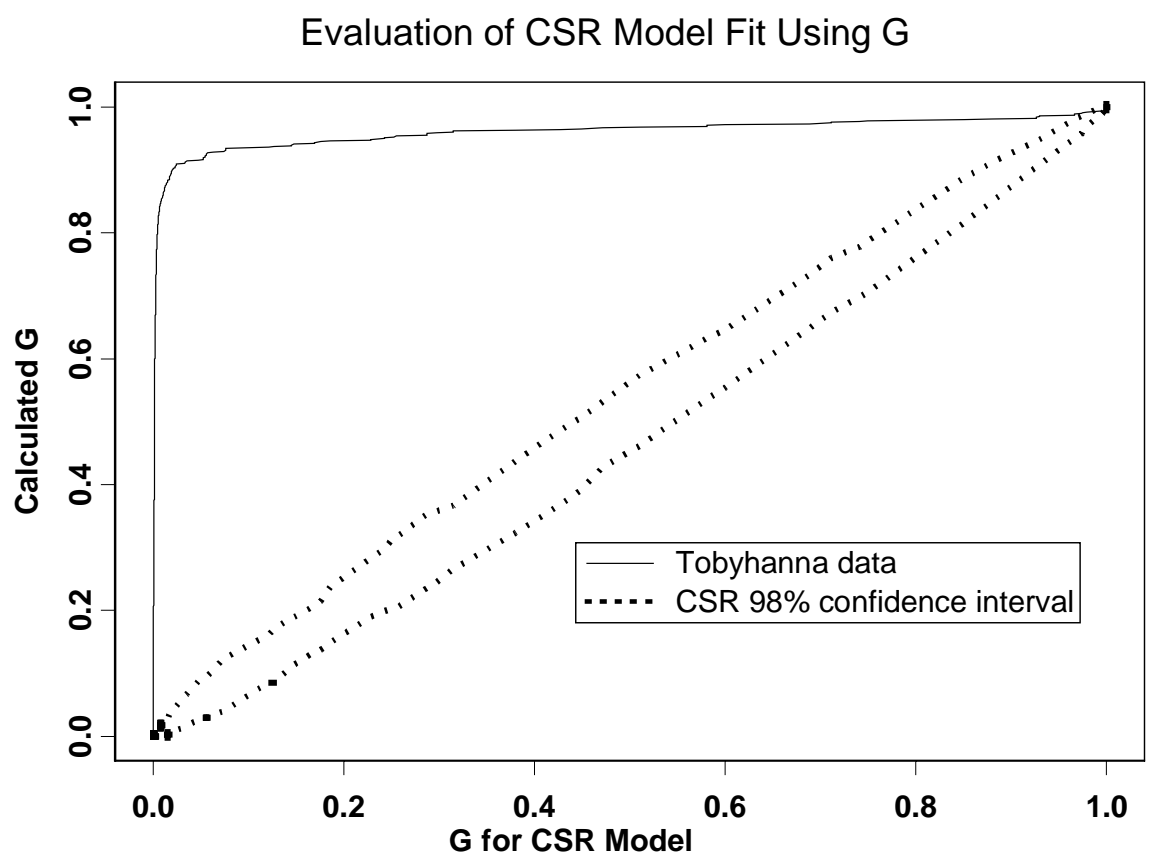

\section{Evaluation of Cluster Model Fit Using G}

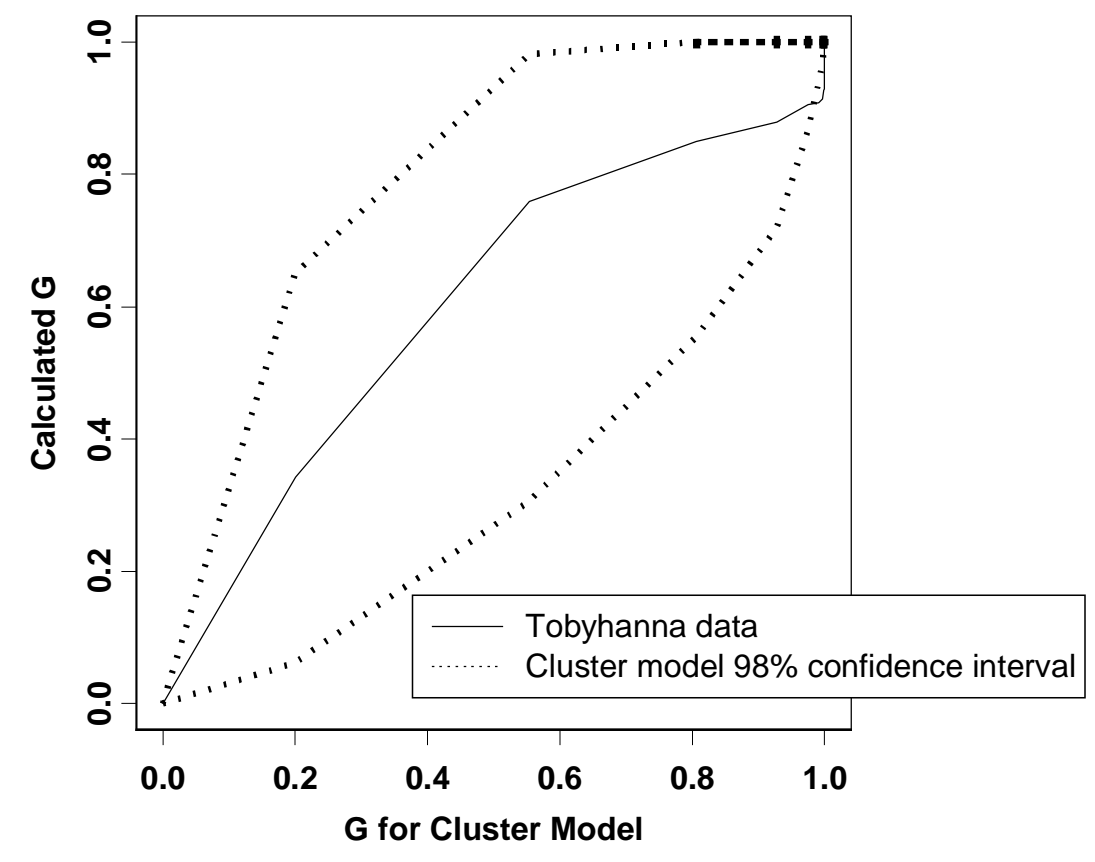

Figure S-3: Comparison of CSR (upper graph) and cluster (lower graph) model fits to the Tobyhanna data using $G$. The cluster model fits the data well, while the data are outside the $98 \%$ confidence interval for the CSR model. 

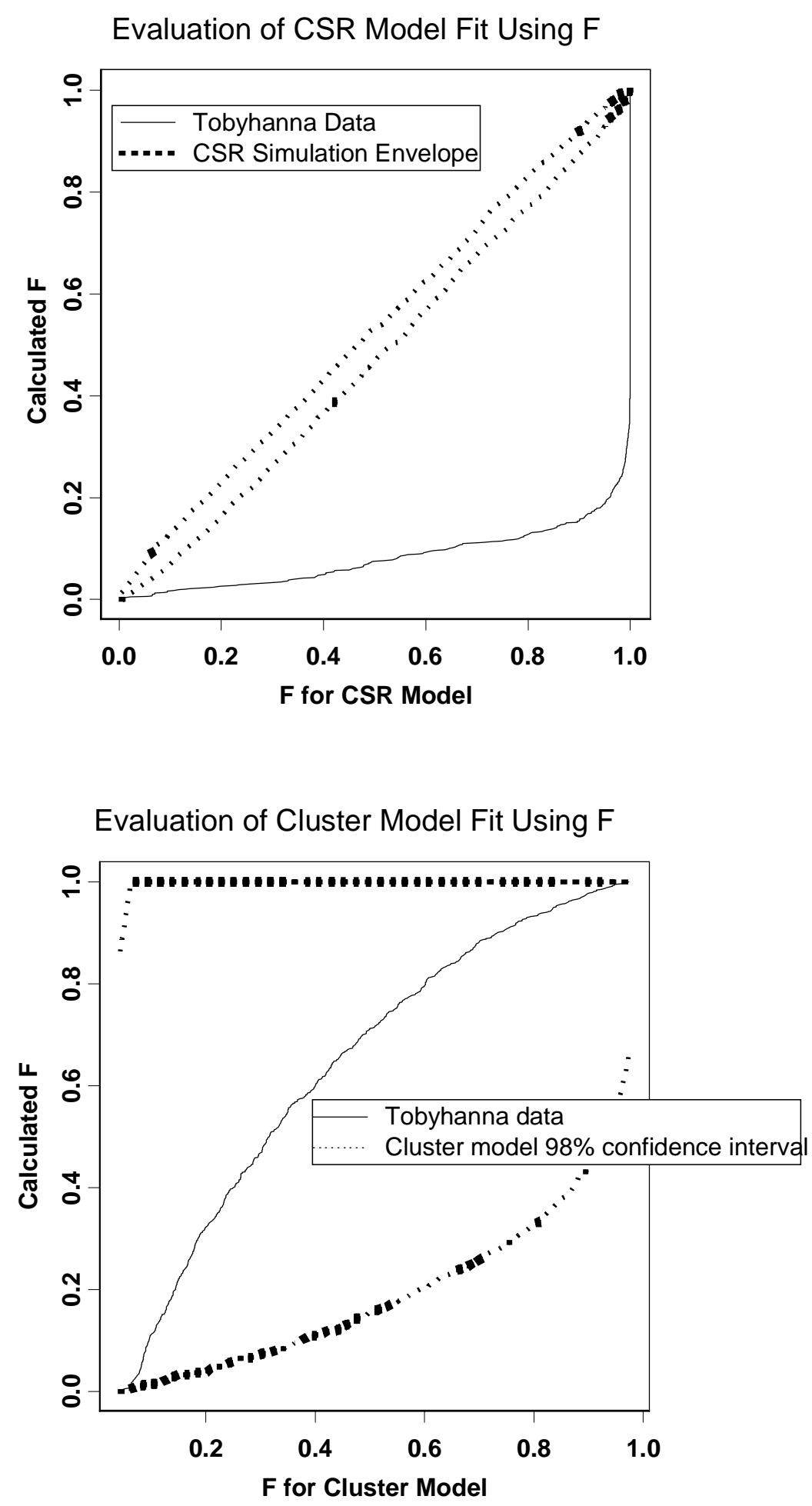

Figure S-4: Comparison of CSR (upper graph) and cluster (lower graph) model fits to the Tobyhanna data using $F$. The data are within the $98 \%$ confidence interval for the cluster model but outside of it for the CSR model. 